\title{
Applied Force during Vitreoretinal Microsurgery with Handheld Instruments
}

\author{
Anirudha D. Jagtap, Cameron N. Riviere \\ The Robotics Institute, Carnegie Mellon University, Pittsburgh, PA, USA
}

\begin{abstract}
Applied force was measured in vivo during vitreoretinal surgery in rabbits, in three types of task: membrane peeling, vessel puncture/cannulation, and vessel dissection. Quantitative results are presented and compared with similar measurements taken in vitro in a porcine retina, in which no scleral interaction is present.
\end{abstract}

Keywords-force sensor, microsurgery

\section{INTRODUCTION}

Microsurgery entails complex manual manipulations that are hampered by the limits of human performance [1]. Methods to overcome the limits of manual positioning accuracy have been the subject of several investigations in recent years, leading to the development of a variety of different types of accuracy-enhancement systems [2-4]. Some of these systems may enable new types of microsurgical procedures in the near future.

The limits of human force perception present another difficulty. Gupta et al. recorded axial tool shaft forces during retinal manipulation in vitro in porcine cadaver eyes and determined that $75 \%$ of all measured forces are below $7.5 \mathrm{mN}$ in magnitude [5]. Their subsequent tests of perception of force showed that only $19 \%$ of events at this force level were felt by the surgeon. These results indicate that much of clinical vitreoretinal microsurgery is being performed solely with visual feedback, a condition that has been shown to be detrimental to performance [6]. Force feedback during microsurgery therefore has the potential to improve both accuracy and task completion time.

In recent years there have been several investigations of force sensing for microsurgery or micromanipulation. Salcudean et al. have presented results from microsurgical experiments in vitro using force-reflecting teleoperation [7], and Tokuyasu and Kitamura have presented in vitro results for reflected feedback of torque [8]. Zhou et al. have presented similar results in non-surgical microassembly tasks [9]. Several other investigators have reported results from in vitro sensing of force in microsurgical systems $[5,10]$. Not all of these sensors provide three-axis sensing, however, and some of them are not of suitable size for incorporation within a handheld vitreoretinal microsurgical instrument. As a result, Berkelman et al. [11] designed a triaxial force sensor specifically for mounting within such

Funding provided by National Science Foundation (grant no. EEC9731748) and National Institutes of Health (grant no. 1 R01 EB000526). an instrument. This sensor is a double cross design with eight strain gauges connected in four half bridges.

This paper presents a study of applied tool tip force during vitreoretinal microsurgery in vivo, to verify the results in vitro reported in [5], using the sensor presented in [11]. The study is also a step in the development of a force feedback system usable in clinical microsurgery with handheld instruments.

\section{METHODOLOGY}

The sensor presented in [11] was recalibrated and used for this study. It was fitted to a handheld microsurgical instrument as shown in Fig. 1. The strain gauge bridge outputs were connected to signal conditioner/amplifiers (S7DC Transducer Amplifier, RDP Group, Wolverhampton, U.K.). The four amplifier outputs were digitized at $100 \mathrm{~Hz}$.

Vitreoretinal microsurgery was performed in rabbits at the Doheny Eye Institute of the University of Southern California. Tests were performed in a total of five eyes in three rabbits. Three types of tasks were performed:

- Peeling of the internal limiting membrane using a bent needle tip ( $\mathrm{N}=60$ discrete events);

- Puncture of retinal vessels using a micropipette $(\mathrm{N}=20)$;

- Dissection of vessels (separating them from the retina) using a spatula-type pick $(\mathrm{N}=44)$.

Between 30 and 60 minutes of data were recorded from each eye. Each procedure was videotaped through the operating microscope. The video and force recordings were synchronized. Data were recorded and processed in three dimensions: $x, y$, and $z$ (with $z$ being the long axis of the instrument). After visual inspection of data files to identify the approximate frequency content of the manipulation events using the instrument tip, data were highpass filtered to isolate these events from other measured force components apparently stemming from scleral contact. The filter was an eighth-order Butterworth highpass filter with a cutoff frequency of $0.1 \mathrm{~Hz}$. Using the synchronized video, the specific segments of the force recording in which the tasks of interest were performed were extracted from the overall recording for purposes of data analysis. These highpass-filtered segments were then concatenated to make one data set for each of the three tasks. The root mean square (rms) amplitude and the total range was computed for each axis of each data set.

The same procedure, including video recording, was repeated in vitro with an isolated cadaver pig retina for the vessel puncture $(\mathrm{N}=20)$ and dissection $(\mathrm{N}=9)$ tasks. Fig. 2 displays an image from the video recording. There was no 
sclerotomy in the pig retina test; the anterior portion of the eye was excised.

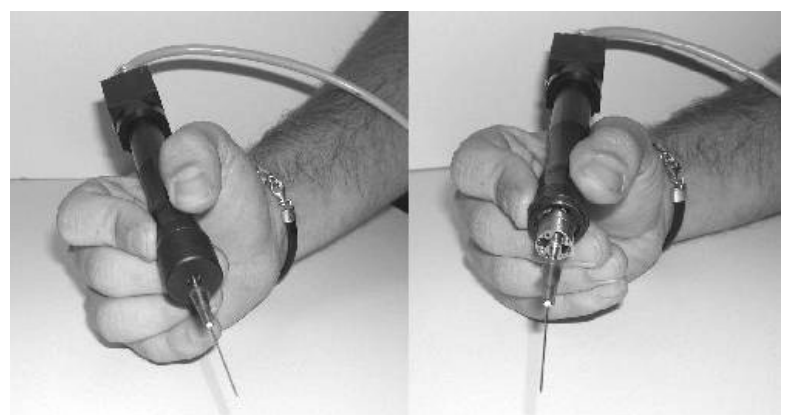

Fig. 1. The surgical instrument fitted with the triaxial force sensor. (a) As used in surgery, with the end cap over the force sensor. (b) With the end cap removed, showing the tip force sensor.

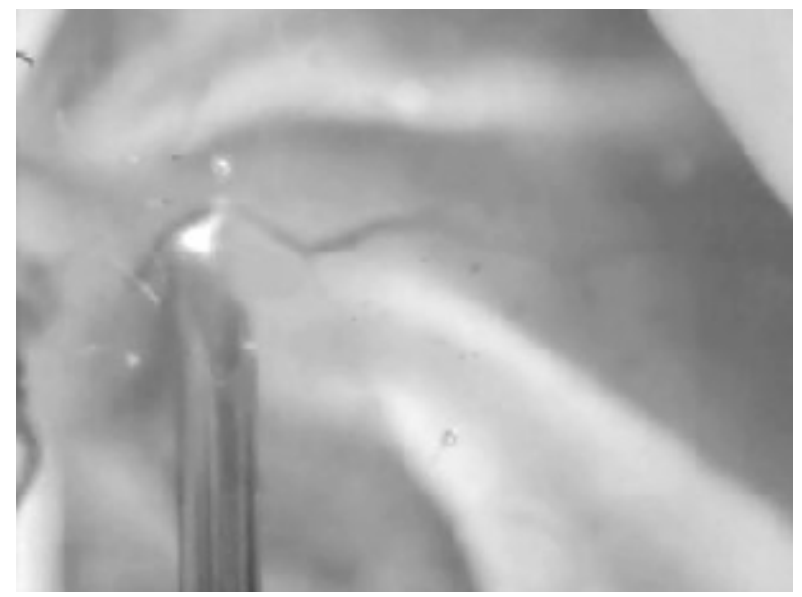

Fig. 2. Retinal surgery in vitro in a porcine retina using the force sensor. The needle in the left half of the image is contacting a retinal vessel, which can be seen as a dark wavy line stretching from the needle tip toward the right side of the image.

\section{RESULTS}

Table I presents the rms amplitude and maximum amplitude as estimated for membrane peeling in vivo in rabbits. Table II presents rms amplitude and maximum amplitude for retinal vessel puncture in vivo. Table III contains the rms and maximum amplitude results for retinal vessel dissection in vivo. Figs. 3-5 display the $y$ coordinate of the filtered concatenated data sets from which the data in these tables are obtained.

Tables IV and $\mathrm{V}$ show the corresponding results for vessel puncture and dissection, respectively, in vitro in the porcine retina.
TABLE I

Measured Force during MEMBrane PeEling in Vivo

\begin{tabular}{ccc}
\hline Direction & RMS $(\mathrm{mN})$ & $\begin{array}{c}\text { Range } \\
(\mathrm{mN})\end{array}$ \\
\hline $\mathrm{X}$ & 56 & 501 \\
$\mathrm{Y}$ & 54 & 463 \\
$\mathrm{Z}$ & 357 & 3142
\end{tabular}

TABLE II

MeAsured Force DURING Vessel PunCtuRE IN VIVO

\begin{tabular}{ccc}
\hline Direction & RMS $(\mathrm{mN})$ & $\begin{array}{c}\text { Range } \\
(\mathrm{mN})\end{array}$ \\
\hline $\mathrm{X}$ & 24 & 161 \\
$\mathrm{Y}$ & 27 & 171 \\
$\mathrm{Z}$ & 74 & 486
\end{tabular}

TABLE III

MEASURED ForCE DURING VESSEL DISSECTION IN VIVO

\begin{tabular}{ccc}
\hline Direction & RMS $(\mathrm{mN})$ & $\begin{array}{c}\text { Range } \\
(\mathrm{mN})\end{array}$ \\
\hline $\mathrm{X}$ & 25 & 217 \\
$\mathrm{Y}$ & 15 & 124 \\
$\mathrm{Z}$ & 575 & 5877
\end{tabular}

TABLE IV

MEASURED ForCE DURING VeSSEl PUNCTURE IN VitRo

\begin{tabular}{ccc}
\hline Direction & RMS $(\mathrm{mN})$ & $\begin{array}{c}\text { Range } \\
(\mathrm{mN})\end{array}$ \\
\hline $\mathrm{X}$ & 13 & 111 \\
$\mathrm{Y}$ & 8 & 81 \\
$\mathrm{Z}$ & 20 & 181
\end{tabular}

TABLE V

MEASURED ForCE DURING VESSEL DisSECTION IN VITRO

\begin{tabular}{ccc}
\hline Direction & RMS $(\mathrm{mN})$ & $\begin{array}{c}\text { Range } \\
(\mathrm{mN})\end{array}$ \\
\hline $\mathrm{X}$ & 1 & 25 \\
$\mathrm{Y}$ & 1 & 16 \\
$\mathrm{Z}$ & 67 & 82
\end{tabular}

\section{DISCUSSION}

The membrane peeling task performed here is similar in nature to epiretinal membrane peel procedures that are frequently performed, and it is expected that the forces recorded are similar. The vessel puncture task is comparable to the manipulation that is necessary to perform retinal vein cannulation. The forces measured during vessel dissection or separation from the retina are expected to be similar to those that would be applied during arteriovenous sheathotomy.

The force values obtained for the porcine cadaver retina are comparable to those reported in [5], though perhaps slightly higher. The forces obtained in vivo in rabbits are considerably higher. This would appear to be due to contact with the sclerotomy. It seems likely that discrimination 
between forces applied at the tool tip and forces due to contact with the sclera seems likely to be a significant challenge in the development of useful force feedback for vitreoretinal microsurgery.

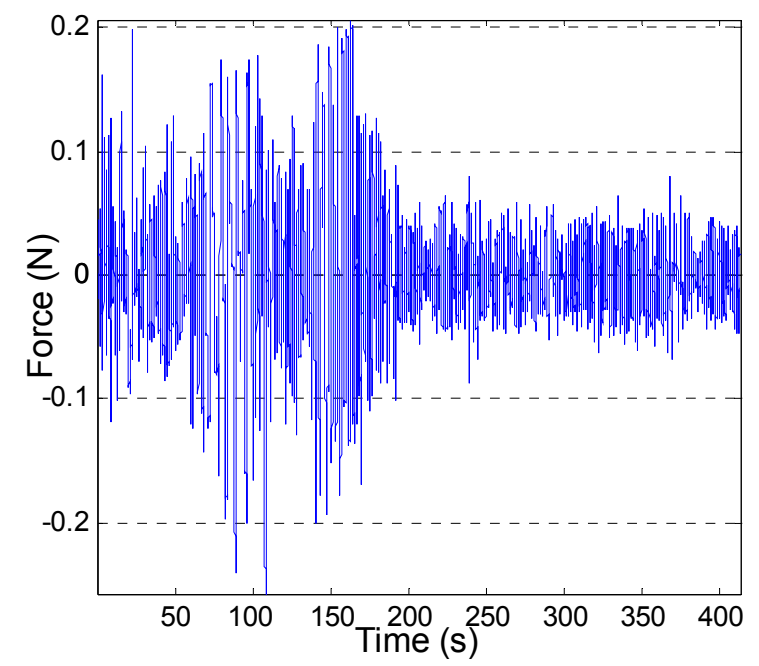

Fig. 3. The filtered concatenated data set for membrane peeling in vivo in rabbits, $y$ coordinate.

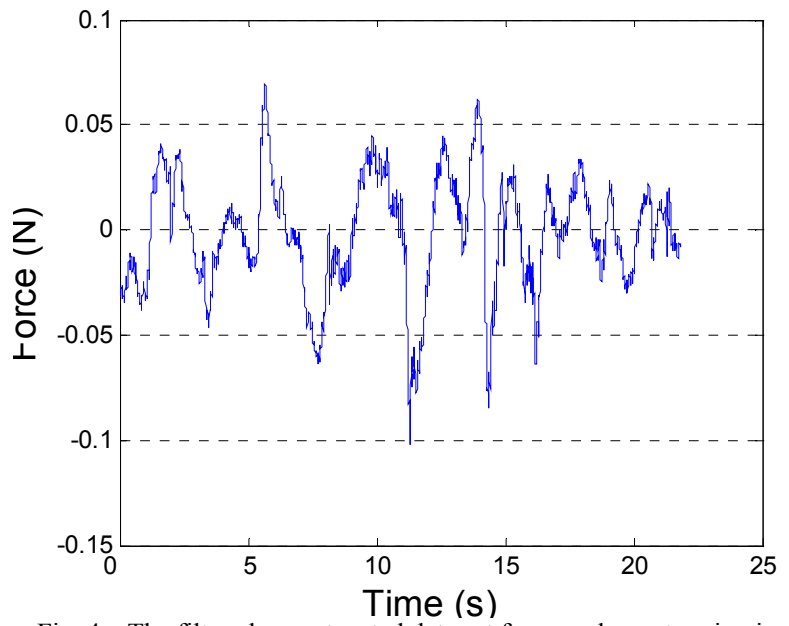

Fig. 4. The filtered concatenated data set for vessel puncture in vivo in rabbits, $y$ coordinate.

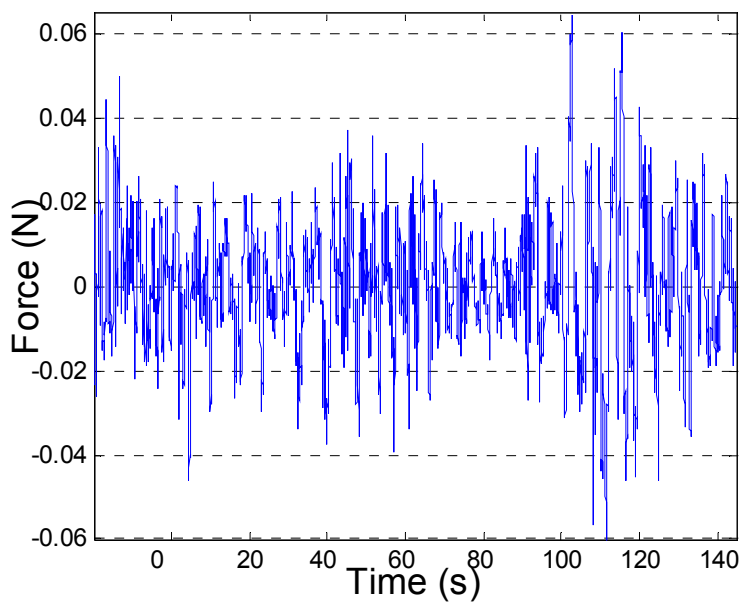

Fig. 5. The filtered concatenated data set for vessel dissection in vivo in rabbits, $y$ coordinate.

\section{REFERENCES}

[1] S. Tamai, "History of microsurgery-from the beginning until the end of the 1970s," Microsurgery, vol. 14, no. 1, pp. 6-13, 1993.

[2] H. Das, H. Zak, J. Johnson, J. Crouch, and D. Frambach, "Evaluation of a telerobotic system to assist surgeons in microsurgery," Computer Aided Surg., vol. 4, pp. 15-25, 1999.

[3] R. Taylor, P. Jensen, L. Whitcomb, A. Barnes, R. Kumar, D. Stoianovici, P. Gupta, Z. Wang, E. de Juan, Jr., and L. Kavoussi, "A steady-hand robotic system for microsurgical augmentation," Int. J. Robot. Res., vol. 18, pp. 1201-1210, 1999.

[4] C. N. Riviere, W. T. Ang, and P. K. Khosla, "Toward active tremor canceling in handheld microsurgical instruments," IEEE Trans. Rob. Autom., vol. 19, pp. 793-800, 2003.

[5] P.K. Gupta, P.S. Jensen, and E. de Juan, Jr., "Surgical forces and tactile perception during retinal microsurgery," in C. Taylor and A. Colchester, eds., MICCAI'99: Medical Image Computing and Computer-Assisted Intervention, pp. 1218-1225, Cambridge, England, 1999. Springer-Verlag.

[6] D.A. Kontarinis and R.D. Howe, "Tactile display of vibratory information in teleoperation and virtual environments," Presence, vol. 4, no. 4, pp. 387-402, 1995.

[7] S. E. Salcudean, S. Ku, and G. Bell, "Performance measurement in scaled teleoperation for microsurgery," Proc. CVRMedMRCAS'97, Lect. Notes Comput. Sci., vol. 1205, pp.789-798, 1997.

[8] T. Tokuyasu and T. Kitamura. A study on minute torque transmission for tele microsurgery. Proc. 2001 JSME Conf. Robotics and Mechatronics (ROBOMEC'01), Takamatsu, Japan, June 2001 (in Japanese).

[9] Y. Zhou, B. J. Nelson, and B. Vikramaditya, "Fusing force and vision feedback for micromanipulation," Proc. IEEE Int. Conf. Robot. Autom., Leuven, Belgium, May 1998, pp. 1220-1225

[10] A. Menciassi, A. Eisinberg, G. Scalari, C. Anticoli, M.C. Carrozza, P. Dario. Force feedback-based microinstrument for measuring tissue properties and pulse in microsurgery. Proc. IEEE Int. Conf. Robotics \& Automation (ICRA2001), Seoul, Korea, May 21-26, 2001, pp. 626-631.

[11] P. J. Berkelman, L. L. Whitcomb, R. H. Taylor, and P. Jensen, "A miniature microsurgical instrument tip force sensor for enhanced force feedback during robot-assisted manipulation," IEEE Trans. Rob. Autom., vol. 19, no. 5, pp. 917-921, 2003. 